\title{
New and Advanced Numerical Strategies for the Simulation of Material Forming
}

\author{
Francisco Chinesta $^{1}$, Thierry Coupez ${ }^{2}$, and Elias Cueto ${ }^{3}$ \\ 1 LMSP, UMR CNRS-ENSAM, 151 Boulevard de l'Hopital, F-75013 Paris, France \\ francisco.chinesta@paris.ensam.fr, \\ 2 CEMEF, UMR CNRS-ENSMP, BP 207, F-06904 Sophia-Antipolis Cedex, France \\ Thierry.Coupez@ensmp.fr, \\ 3 I3A, University of Zaragoza, Maria de Luna 3, E-50018 Zaragoza, Spain \\ ecueto@unizar.es
}

\begin{abstract}
In recent years new and advanced numerical strategies have opened new possibilities in the simulation of forming processes. Multiscale descriptions, meshless methods and enhanced finite element approaches are some techniques that have contributed to the enhancement of forming process simulations. These approaches will be revisited in this chapter.
\end{abstract}

\section{Microscopic approaches}

\subsection{Solid mechanics framework}

Materials can be described at different scales. The finest level of description consists of the atomic level and the coarsest one concerns the scale at which the conformed part is defined. Molecular dynamics simulation works at the atomic level and allows to account for complex physics in a simple and natural way. Thus, knowing at a certain time the position of the atoms, the resultant force applying at each atom can be easily computed from a semi-empirical atomic potential, and from it the atoms acceleration computed and the velocities and atomic positions updated. The main drawback of this approach lies in the extremely large computing time required to perform realistic simulations even when small domains and time periods are considered,as well as the semi-empirical interatomic potentials usually considered in such approaches.

The establishment of more accurate atomic potentials require the solution of the Schrodinger equation in the quantum mechanics framework, whose main difficulty lies in the curse of dimensionality that we consider later, and that constitutes today a real challenge. In recent years molecular dynamics approaches were considered in the framework of forming processes involving cutting [39] or contact with friction 12], both presented during the recent Esaform conferences.

The micro-macro approach when both descriptions coexist in the physical space as well as the definition of efficient bridges between both descriptions defined in contiguous regions are topics in active development nowadays 24] [43] [23]. 
In an intermediate scale other approach based on the discrete finite element was successfully applied for treating granular media, being [40] or [21] some examples of works presented during the Esaform conferences in recent years.

\subsection{Fluid mechanics framework}

This section concerns the liquid state of different materials involved in forming processes. In some cases, as in casting, the resulting constitutive equation of involved materials results simple, but the high Reynolds number involved in the forming processes induce numerous numerical difficulties. For other kind of materials, the ones involving microstructure, the main difficulty is coming from the inherent multiscale character of its mechanical behavior. This section focuses on this complex fluids (polymer melts or particle suspensions).

Many natural and synthetic fluids are viscoelastic materials, in the sense that the stress endured by a macroscopic fluid element depends upon the history of the deformation experienced by that element. Notable examples include polymer solutions and melts, liquid crystalline polymers and fibre suspensions. Rheologists thus face a challenging non-linear coupling between flow-induced evolution of molecular configurations, macroscopic rheological response, flow parameters (such as the geometry and boundary conditions) and final properties. Theoretical modelling and methods of computational rheology have an important role to play in elucidating this coupling.

Atomistic modelling is the most detailed level of description that can be applied today in rheological studies, using techniques of non equilibrium molecular dynamics. Such calculations require enormous computer resources, and then they are currently limited to flow geometries of molecular dimensions. Consideration of macroscopic flows found in processing applications calls for less detailed mesoscopic models, such as those of kinetic theory.

Kinetic theory models can be very complicated mathematical objects. It is usually not easy to compute their rheological response in rheometric flows, and their use in numerical simulations of complex flows has long been thought impossible. The traditional approach has been to derive from a particular kinetic theory model a macroscopic constitutive equation that relates the viscoelastic stress to the deformation history. The majority of constitutive equations used in continuum numerical simulations are indeed derived (or at least very much inspired) from kinetic theory. Indeed, derivation of a constitutive equation from a model of kinetic theory usually involves closure approximations of a purely mathematical nature such as decoupling or pre-averaging. It is now widely accepted that closure approximations have a significant impact on rheological predictions for dilute polymer, solutions, or fiber suspensions.

Since the early 1990's the field has developed considerably following the introduction of the CONNFFESSIT method by Ottinger and Laso [37]. Kinetic theory provides two basic building blocks: the diffusion or Fokker-Planck equation that governs the evolution of the distribution function (giving the probability distribution of configurations) and an expression relating the viscoelastic 
stress to the distribution function. The Fokker-Planck equation has the general form:

$$
\frac{d \psi}{d t}+\frac{\partial}{\partial \mathbf{X}}(\mathbf{A} \psi)=\frac{\partial}{\partial \mathbf{X}}\left(\mathbf{D} \frac{\partial \psi}{\partial \mathbf{x}}\right)
$$

where $\frac{d \psi}{d t}$ is the material derivative, vector $\mathbf{X}$ defines the coarse-grained configuration and has dimensions $N$. Factor $\mathbf{A}$ is a $N$-dimensional vector that defines the drift or deterministic component of the molecular model. Finally $\mathbf{D}$ is a symmetric, positive definite $N \times N$ matrix that embodies the diffusive or stochastic component of molecular model. In general both $\mathbf{A}$ and $\mathbf{D}$ (and in consequence the distribution function $\psi$ ) depend on the physical coordinates $\mathbf{x}$, on the configuration coordinates $\mathbf{X}$ and on the time $t$.

The second building block of a kinetic theory model is an expression relating the distribution function and the stress. It takes the form:

$$
\tau_{p}=\int_{C} \mathbf{g}(\mathbf{X}) \psi d \mathbf{X}
$$

where $C$ represents the configuration space and $\mathbf{g}()$ is a model-dependent tensorial function of configuration. In a complex flow, the velocity field is a priori unknown and stress fields are coupled through the conservation laws. In the isothermal and incompressible case the conservation of mass and momentum balance are then expressed (neglecting the body forces) by:

$$
\left\{\begin{array}{l}
\nabla \cdot \mathbf{v}=0 \\
\rho \frac{d \mathbf{v}}{d t}=\nabla \cdot\left(-p \mathbf{I}+\tau_{p}+\eta_{s} \mathbf{d}\right)
\end{array}\right.
$$

where $\rho$ is the fluid density, $p$ the pressure and $\eta_{s} \mathbf{d}$ a purely viscous component. The set of coupled equations (11)-(3i), supplemented with suitable initial and boundary conditions in both physical and configuration spaces, is the generic multiscale formulation. Three basic approaches have been adopted for exploiting the generic multiscale model:

1. The continuum approach wherein a constitutive equation of continuum mechanics that relates the viscoelastic stress to the deformation history is derived from, and replaces altogether, the kinetic theory model (1) and (2). The derivation process usually involves closure approximations. The resulting constitutive model takes the form of a differential, integral or integrodifferential equation.

2. The Fokker-Planck approach wherein one solves the generic problem (1) to (3) as such, in both configuration and physical spaces. The distribution function is thus computed explicitly as a solution of the Fokker-Planck equation (11). The viscoelastic stress is computed from (2).

3. The Stochastic approach which draws on the mathematical equivalence between the Fokker-Planck equation (酉) and the following Ito stochastic differential equation:

$$
\mathbf{d} \mathbf{X}=\mathbf{A} d t+\mathbf{B} \mathbf{d} \mathbf{W}
$$


where $\mathbf{D}=\mathbf{B} \mathbf{B}^{T}$ and $\mathbf{W}$ is a Wiener stochastic process of dimension $N$. In a complex flow, the stochastic differential equation (4) applies along individual flow trajectories, the time derivative is thus a material derivation. Instead of solving the deterministic Fokker-Planck equation (11), one solves the associated stochastic differential equation (国) for a large ensemble of realizations of the stochastic process $\mathbf{X}$ by means of a suitable numerical technique. The distribution function is not computed explicitly, and the viscoelastic stress (22) is readily obtained as an ensemble average.

The control of the statistical noise is a major issue in stochastic micro-macro simulations based on the stochastic approach (for more details concerning the micro-macro approach reader can refers to the excellent review paper 28] and the references therein). Some stochastic simulations of muti-bead-spring (MBS) models have been successfully carried out, see for example 44. These problems do not arise at all in the Fokker-Planck approach. The difficulty, however, is that the Fokker-Planck equation (11) must be solved for the distribution function in both physical and configuration spaces. This necessitates a suitable discretization procedure for all relevant variables, namely position $\mathbf{x}$, configuration $\mathbf{X}$ and time $t$. Until now, the dimensionality of the problem could be daunting and consideration of molecular models with many configurational degrees of freedom did not appear feasible. This probably explains why relatively few studies based of the Fokker-Planck approach have appeared in the literature until very recently at least. In [14] [30] the resolution of the Fokker-Planck equation involving a moderate number of dimensions is considered. Another deterministic particle approach, very close to that proposed in [13], was analyzed in [3] using the SPH meshless approach.

An appealing strategy that allows alleviating the computational effort is based on the use of reduced approximation bases obtained by applying the Karhunen-Loève decomposition, succesfully applied in complex fluid simulation in 41] and [2], however high dimensional models are out of its applicability, because in this case the definition of a mesh results simply prohibitory.

Some attempts exist concerning the treatment of multidimensional problems. The interested reader can refer to [11] for a review on sparse grids methods involving sparse tensor product spaces, but despite of its optimality, the interpolation is defined in the whole multidimensional domain, and consequently only problems defined in spaces of dimension of the order of tens can be treated [1]. In [10] multidimensional problems are revisited and deeply analyzed, and for this purpose new mathematical entities are introduced. In [4] we considered the steady state solution of some classes of multidimensional partial differential equations by using a separated representation. In [5] this technique was extended for solving accurately and efficiently multidimensional transient kinetic theory models.

Some works focussing in the solution of the multidimensional Fokker-Planck equation defining the micro-macro description were presented during the recent Esaform conferences (see for example [6]) however these models only concerned simple rheological flows. The real challenge for the next 10 years will be the exten- 
sion of these procedures for treating the complex flows encountered in polymer forming processes.

\section{Meshless methods: the possibility for Lagrangian simulations and much more}

\subsection{Introduction}

It is not easy to explain what a meshless method is in few words. Since the meshless irruption after the pioneer work by Touzot and Villon [36], many different meshless methods have arisen, with many different characteristics and names. Just to cite a few, the Element-Free Galerkin [9], the Reproducing Kernel Particle Method [29], the Natural Element Method [46] 20], or the Generalized Finite Element Method [45], are examples of these different methods. They are based either in Galerkin or collocation approaches, employ different kinds of approximation for the essential variables (moving least squares, natural neighbour approximants, wavelets, etc.) and also employ —when talking about Galerkin strategies - different numerical integration procedures.

What is essential then in a numerical method to be considered as meshless is its ability to maintain the accuracy despite the distortion of the mesh (or, more properly, the cloud of points). It is well-known that the Finite Element method suffers from lack of accuracy if the mesh becomes distorted [7]. Meshless methods, however, adapt the connectivity of the "elements" as the cloud of points evolves, in a process transparent to the user. Many meshless methods use radiallysupported shape functions (or employ tensor-product shape functions, thus giving a rectangular support), see Fig. 1. Thus, the connectivity of each element (defined as the list of nodes in-

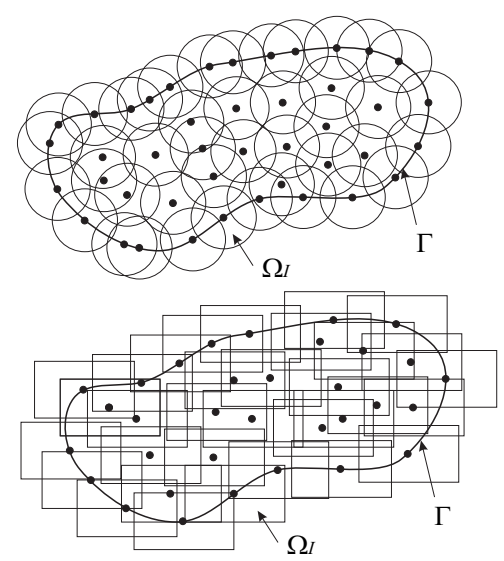

Fig. 1. Covering of a two-dimensional domain $\Omega$ by the shape functions' support, $\Omega_{I}$.

fluencing the portion of the space in which numerical integration is to be performed) changes as the cloud evolves.

Element-Free Galerkin methods (EFGM) fall within the class of meshless methods that employ cobertures of the domain such as that in Fig. 1. In this case, EFGM employ a Galerkin perspective and Moving Least Squares interpolation to construct shape functions with arbitrary degree of reproducibility. Reproducing Kernel Particle Methods, although originally developed from Smooth Particle Hydrodynamics approaches, are entirely equivalent to EFGM.

However, many meshless methods with radially-supported shape functions lack of an appropriate interpolation along the boundaries (which is readily seen 
from Fig. 1 if we note that interior nodes influence on the boundary values). This is a particularly important problem in the simulation of forming processes, where issues related to friction, for instance, are frequently noteworthy. The Natural Element Method (NEM) [46] [20] solves this problem in a very elegant way, by utilizing natural neighbour interpolation, instead of Moving Least Squares, in a Galerkin framework. Natural neighbour interpolation functions do not have circular support (instead, they cover the union of circumcircles of each Delaunay triangle containing the node) and this fact makes possible the exact imposition of essential boundary conditions, up to the degree of consistency of the method.

Other, similar, methods, that also employ natural neighbour-based interpolants have been developed in recent years and applied to the simulation of forming processes, see, for instance, [25] [26].

Many other meshless methods exist, based on collocation as well as Galerkin approaches, but probably the EFGM and the NEM have been the most popular ones within the forming processes community.

\subsection{Application of meshless methods to the simulation of forming processes.}

The fact that meshless methods do not lack accuracy as mesh distorts opens the possibility to perform Lagrangian simulations instead of Eulerian or Arbitrary Lagrangian-Eulerian ones, which had been the most employed ones. This is especially challenging in fields where traditional Finite Element procedures fail or present difficulties. Some examples developed by the authors follow. They are only included intending show how, qualitatively, meshless methods can help in the simulation of very complex forming processes.

Simulation of injection moulding of short fiber reinforced thermoplastics. Free-surface flows is a typical example of this kind of problems. The location of the free surface had been traditionally done by means of Volume of Fluid (VoF) or similar techniques, in which a variable representing the portion of an Eulerian element which is filled by a liquid must be advected with the material velocities.

Mechanical modelling of short fibers suspensions flows is usually achieved in the framework of dilute or semi-dilute suspensions of non-spherical particles in a Newtonian fluid. The resulting system of equations involves the coupling of an elliptic problem with an advection problem related to the fluid history. The elliptic problem is associated with the equations of motion whereas the advection equation describes the time evolution of the anisotropic viscosity tensor (fiber orientation) or more generally the microstructural state. The second problem presents two difficulties: it is non-linear and hyperbolic.

Coupled models take into account both the dependence of the kinematics with the fiber orientation and the orientation induced by the flow kinematics. Usually the coupled models are solved by means of a fixed point strategy. In this

case, at each iteration the flow kinematics results from the solution of motion and 


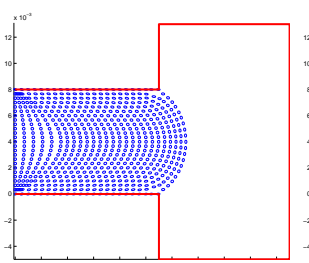

(a)

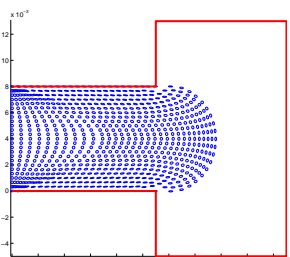

(b)

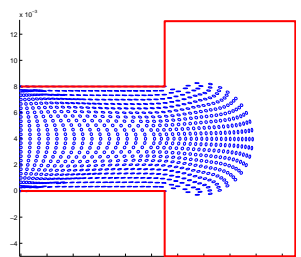

(c)

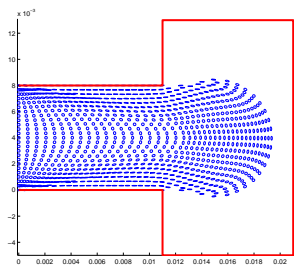

(d)

Fig. 2. Evolution of the orientation field in the simulation of an injection moulding.

mass conservation equations, using the fiber orientation field from the previous iteration. From the kinematics just computed, the fiber orientation is updated solving the advection equation governing its evolution. In Fig. 2 four snapshots of the evolution of the orientation field are shown. The orientation is represented by elipses indicating the probability of finding a fiber oriented in each direction.

In [31 a deeper insight on the constitutive modelling of such flows can be found. The accuracy in the numerical treatment of the free surface flow is also noteworthy 32 .

Simulation of orthogonal cutting. One of the very first applications of the NEM to the field of forming processes was made towards the simulation of cutting [17]. The extremely large deformations appearing in such a process make meshless methods an interesting approach to be considered.

Essentially, in this first applications a very simple viscoplasticity model based on a Norton-Hoff law and very simple contact detection algorithms, that considered rigid tool surfaces, was employed. The main purpose was, however, to demonstrate that such a method can easily suffer these high lev-

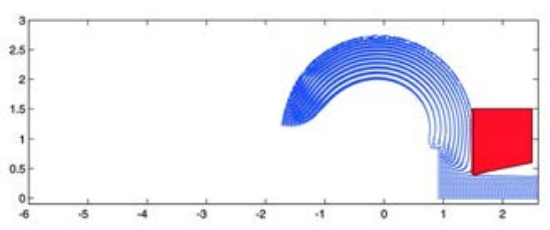
els of strain without lack of accuracy, see Fig. 3.

Three-dimensional simulation of the extrusion of a cross-shaped profile. In order to show the capabilities of the technique presented before, we analyze now the simulation of a cross-shaped aluminium profile.

Nodes located on the upper side of the billet were forced to move with a speed of $2 \frac{\mathrm{mm}}{\mathrm{s}}$, in order to obtain an exit velocity of $1 \frac{\mathrm{m}}{\mathrm{min}}$ approximately. Initially, slipping boundary conditions were considered between the billet and the die and the container. The initial temperature was set to $723 K$. The whole model was 
considered adiabatic, including the profile surface in contact with air. In this case a rigid-plastic Sellars-Tegart material model was used. The simulation ran over 42 time steps of $0.025 \mathrm{~s}$. The obtained evolution for the equivalent strain rate is depicted in Fig. 1 .
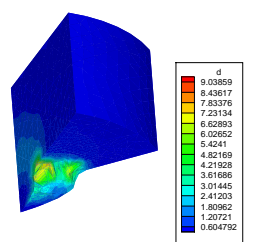

(a) 10th time step.

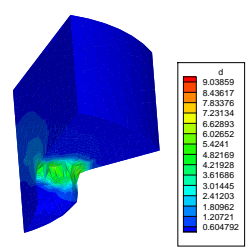

(b) 20th time step.

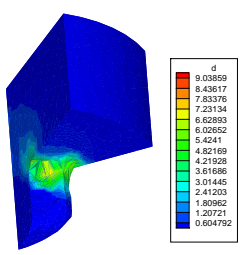

(c) 30th time step.

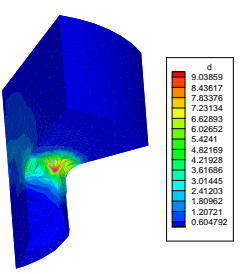

(d) 40th time step.

Fig. 4. Equivalent strain rate $\left(s^{-1}\right)$ for the extrusion simulation.

\subsection{Meshless methods in the ESAFORM conferences: past, present and future}

These examples are intended for suggesting only the wide range of potential application that meshless methods can have in the field of forming processes. But the applications are by no means limited to the before presented. Other examples include the simulation of expanding foams 16], Stefan problems, etc.

In all these cases, meshless methods opened the possibility for a Lagrangian procedure, which is in some cases a very convenient way of overcoming the before-mentioned problems of numerical diffusion in the results due to extensive remeshing.

Concerning the ESAFORM life, meshless methods have been present, up to our knowledge, since the conference held in Liege in 2001 [33] and their contribution extends up to the ninth ESAFORM conference's scientific prize plenary lecture [19]. In between, many papers have been presented in ESAFORM conferences employing meshless method to simulate forming processes. For instance, there is a very active group in Portugal applying these methods to the simulation of various forming proceses [51]. Applications include forging, sheet metal forming, etc. Other contributions include applications of the so-called eXtended Finite Element Method (X-FEM) [22] [35], or the excellent keynote given by N. Sukumar at Salerno in 2003 477. Concerning RKP methods, Joyot has made some interesting contributions along the years, see for instance [27] and references therein. Of course, Prof. Villon has been one of the most active researchers in this field and has presented many works on the topic along the years, see for instance 48].

There remain some interesting challenges concerning meshless methods (both within and out of ESAFORM life). There is a more or less unanimous opinion 
that the issue of numerical integration is crucial in this field, and many groups are now focusing their efforts towards this end. Within ESAFORM life, there remains some problems where meshless methods have much to say. In general, these problems involve very large strains, as mentioned before. Friction stir welding is an example of candidate processes. The extremely large speed of rotation, the coupled thermo-mechanical problem and the extremely large deformations appearing make this process an ideal candidate to benchmark the behaviour of meshless methods.

\section{References}

1. Y. Achdou, O. Pironneau, "Computational methods for option pricing", Siam Frontiers in Applied Mathematics, 2005.

2. A. Ammar, D. Ryckelynck, F. Chinesta, R. Keunings, "On the Reduction of Kinetic Theory Models Related to Finitely Extensible Dumbbells", J. Non-Newtonian Fluid Mech., In press.

3. A. Ammar, F. Chinesta, D. Ryckelynck, "Deterministic particle approach of multibead-spring polymer models", European Journal of Computational Mechanics, In press.

4. A. Ammar, B. Mokdad, F. Chinesta, R. Keunings, "A fast solver for some classes of multidimensional partial differential equations encountered in kinetic theory modelling of complex fluids". Journal of Non-Newtonian Fluid Mechanics, Submitted.

5. A. Ammar, B. Mokdad, F. Chinesta, "A fast solver for some classes of multidimensional partial differential equations encountered in kinetic theory modelling of complex fluids. Part 2: Transient simulation using space-time separated representations". Journal of Non-Newtonian Fluid Mechanics, Submitted.

6. A. Ammar, F. Chinesta, D. Ryckelynck "Deterministic approach of the multi-beadspring polymer models", Proceedings of the 8th Esaform Conference on Material Forming, Cluj-Napoca, Romania.

7. I. Babuška and A. Aziz. On the angle condition in the finite element method. SIAM J. Numer. Anal., 13:214-227, 1976.

8. T. Belytschko, Y. Krongauz, M. Fleming, D. Organ, and W. K. Liu. Smoothing and accelerated computations in the element free galerkin method. Journal of Computational and Applied Mathematics, 1995.

9. T. Belytschko, Y. Y. Lu, and L. Gu. Element-Free Galerkin Methods. International Journal for Numerical Methods in Engineering, 37:229-256, 1994.

10. G. Beylkin, M. Mohlenkamp, "Algorithms for numerical analysis in high dimensions", SIAM J. Sci. Com., 26/6, 2133-2159, 2005.

11. H.J. Bungartz, M. Griebel, "Sparse grids", Acta Numerica, 13, 1-123, 2004.

12. P. Chantrenne, "Atomic scale simulations: use of molecular dynamics", Proceedings of the 9th Esaform Conference on Material Forming, Glasgow, UK, 123-126, 2006.

13. C.V. Chaubal, A. Srinivasan, O. Egecioglu, L.G. Leal, "Smoothed particle hydrodynamics techniques for the solution of kinetic theory problems", J. Non-Newtonian Fluid Mech., 70, 125-154, 1997.

14. C. Chauviere, A. Lozinski, "Simulation of dilute polymer solutions using a FokkerPlanck equation", Computer and Fluids, 33, 687-696, 2004. 
15. J. S. Chen, C. M. O. L. Roque, C. H. Pan, and S. T. Button. Analysis of metal forming process based on meshless method. JOURNAL OF MATERIALS PROCESSING TECHNOLOGY, pages 642-646, 1998.

16. F. Chinesta, E. Cueto, P. Quintela, and J. Paredes. Induced anisotropy of foams forming processes: modelling and simulation. Journal of Materials Processing Technology, 155:1482-1488, 2004.

17. F. Chinesta, Ph. Lorong, D. Ryckelynck, M. A. Martinez, E. Cueto, M. Doblaré, G. Coffignal, M. Touratier, and J. Yvonnet. Thermomechanical cutting model discretisation: Eulerian or Lagrangian, mesh or meshless? International Journal of Forming Processes, 7:83-98, 2004.

18. E. Cueto, M. Doblaré, and L. Gracia. Imposing essential boundary conditions in the Natural Element Method by means of density-scaled $\alpha$-shapes. International Journal for Numerical Methods in Engineering, 49-4:519-546, 2000.

19. E. Cueto. The Natural Element Method for the simulation of forming processes. Plenary conference. Ninth ESAFORM conference on Material Forming, Glasgow, U. K., 2006.

20. E. Cueto, N. Sukumar, B. Calvo, M. A. Martínez, J. Cegoñino, and M. Doblaré. Overview and recent advances in Natural Neighbour Galerkin methods. Archives of Computational Methods in Engineering, 10(4):307-384, 2003.

21. A. Delaplace, C. Rey, "Numerical strategies for describing material failure with discrete element model", Proceedings of the 8th Esaform Conference on Material Forming, Cluj-Napoca, Romania, 101-104, 2005.

22. A. Devan and J. E. Dolbow. An enhanced assumed strain method with discontinuous enrichment. In V. Brucato, editor, ESAFORM Conference on Material Forming, Salerno University, Salerno, Italy, 2003.

23. F. Feyel, "Some application of parallel computing for multiscale and multiphysics problems", Proceedings of the 7th Esaform Conference on Maaterial Forming, Trondheim, Norway, 85-88, 2004.

24. T. Hughes, G. Feijoo, L. Mazzei, J.B. Quincy, "The variational multiscale method - a paradigm for computational mechanics", Computer Methods in Applied Mechanics and Engineering, 166, 3-24, 1998.

25. S. R. Idelsohn, E. Oñate, N. Calvo, and F. del Pin. The meshless finite element method. International Journal for Numerical Methods in Engineering, 58:893-912, 2003.

26. S. R. Idelsohn, E. Oñate, and F. del Pin. The particle finite element method: a powerful tool to solve incompressible flows with free-surfaces and breaking waves. International Journal for Numerical Methods in Engineering, 61 (7):964-989, 2004.

27. P. Joyot, J. Trunzler and F. Chinesta. Accounting for incompressibility in Reproducing Kernel Particle meshless approximations. In A. Rosochowsky, editor, ESAFORM Conference on Material Forming, Strathclyde University, Glasgow, UK, 2006.

28. R. Keunings, "Micro-macro methods for the multiscale simulation viscoelastic flow using molecular models of kinetic theory", Rheology Reviews, D.M. Binding and K. Walters (Edts.), British Society of Rheology, 67-98, 2004.

29. W. K. Liu, S. Jun, S. Li, J. Adee, and T. Belytschko. Reproducing kernel particle methods. International Journal for Numerical Methods in Engineering, 38:16551679, 1995.

30. A. Lozinski, C. Chauviere, "A fast solver for Fokker-Planck equation applied to viscoelastic flows calculations: 2D FENE model", Journal of Computational Physics, 189, 607-625, 2003. 
31. M. A. Martínez, E. Cueto, M. Doblaré, and F. Chinesta. Fixed mesh and meshfree techniques in the numerical simulation of injection processes involving short fiber suspensions. Journal of Non-Newtonian Fluid Mechanics, 115:51-78, 2003.

32. M. A. Martinez, E. Cueto, I. Alfaro, M. Doblare, and F. Chinesta. Updated Lagrangian free surface flow simulations with Natural Neighbour Galerkin methods. International Journal for Numerical Methods in Engineering, 60(13):2105-2129, 2004.

33. M.A. Martinez, E. Cueto, M. Doblare and F. Chinesta. A meshless simulation of injection processes involving short fibers molten composites. In A. M. Habraken, editor, ESAFORM Conference on Material Forming, Université de Liege, Belgium, 2001.

34. N. Moes, J. Dolbow, and T. Belytschko. A Finite Element Method for Crack Growth without Remeshing. International Journal for Numerical Methods in Engineering, 46:131-150, 1999.

35. G. Legrain, N. Moës and E. Verron. Fracture with large deformation using X-FEM. In S. Støren, editor, ESAFORM Conference on Material Forming, Trondheim, Norway, 2004.

36. B. Nayroles, G. Touzot, and P. Villon. Generalizing the finite element method: Diffuse approximation and diffuse elements. Computational Mechanics, 10:307318, 1992.

37. H.C. Öttinger, M. Laso, "Smart polymers in finite element calculation", Int Congr. on Rheology, Brussel, Belguim, 1992.

38. J.A. Sethian. Level Set Methods and Fast Marching Methods Evolving Interfaces in Computational Geometry, Fluid Mechanics, Computer Vision, and Materials Science. Cambridge University Press, 1999.

39. R. Rentsch, "Molecular dynamics simulation of microscopic phenomena in cutting and forming process", Proceedings of the 7th Esaform Conference on Material Forming, Trondheim, Norway, 93-96, 2004.

40. J. Rojek, F. Zarate, C. Agelet de Saracibar, M. Chiumenti, M. Cervera, P.M. Haigh, C. Gilbourne, P. Verdor, "Numerical simulation of sand mould manufacture for lost foam casting process", Proceedings of the 6th Esaform Conference on Material Forming, Salerno, Italy, 655-658, 2003.

41. D. Ryckelynck, F. Chinesta, E. Cueto, A. Ammar, "On the a priori model reduction: overview and recent developments", Archives of Computational Methods in Engineering, 13, 91-128, 2006.

42. D. Ryckelynck, "A priori hyperreduction method: an adaptive approach", J. Computational Physics, 202, 346-366, 2005.

43. V.B. Shenoy, R. Miller, E.B. Tadmor, D. Rodney, R. Phillips, M. Ortiz, "An adaptive finite element approach to atomic scale mechanics - the quasicontinuum methdos", Journal of the Mechanics and Physics of Solids, 47, 611-642, 1999.

44. M. Somasi, B. Khomami, N.J. Woo, J.S. Hur, E.S.G. Shaqfeh, "Brownian dynamics simulations of bead-rod and bead-spring chains: numerical algorithms and coarsegraining issues", J. Non-Newtonian Fluid Mech., 108/1-3, 227-255, 2002.

45. T. Strouboulis, K. Copps, and I. Babuška. The generalized finite element method. Computer Methods in Applied Mechanics and Engineering, 190:4081-4193, 2001.

46. N. Sukumar, B. Moran, and T. Belytschko. The Natural Element Method in Solid Mechanics. International Journal for Numerical Methods in Engineering, 43(5):839-887, 1998.

47. N. Sukumar. Meshless methods and Partition of Unity Finite Elements. In V. Brucato, editor, ESAFORM Conference on Material Forming, Salerno University, Italy, 2003. 
48. D. Chamoret, A. Rassineux, J.-M. Bergheau, P. Villon, Modelling of contact surface by local hermite diffuse interpolation. Proceedings of ESAFORM 2001, The 4th international ESAFORM conference on material forming, Liège, Belgique, 1, p. 179-182, 2001.

49. S. W. Xiong, C. S. Li, J. M. C. Rodrigues, and P. A. F. Martins. Steady and nonsteady state analysis of bulk forming processes by the reproducing kernel particle method. FINITE ELEMENTS IN ANALYSIS AND DESIGN, pages 599-614, 2005.

50. J. Yvonnet, D. Ryckelynck, P. Lorong, and F. Chinesta. A new extension of the Natural Element method for non-convex and discontnuous problems: the Constrained Natural Element method. International Journal for Numerical Methods in Enginering, 60(8):1452-1474, 2004.

51. Cai Zheng and J. M. A. Cesar de Sa. A meshless method application on the localization phenomena of ductile damage in metal forming. In A. Rosochowsky, editor, ESAFORM Conference on Material Forming, Strathclyde University, Glasgow, UK, 2006.

52. J. Zhou, L. Li, and J. Duszczyk. 3D FEM simulation of the whole cycle of aluminium extrusion throughout the transient state and the steady state using the updated Lagrangian approach. Journal of Materials Processing Technology, 134:383397, 2003. 